\title{
Postcricoid Carcinoma
}

National Cancer Institute

\section{Source}

National Cancer Institute. Postcricoid Carcinoma. NCI Thesaurus. Code C8595.

A carcinoma of the hypopharynx that arises from the postcricoid region. 